\title{
Exploring the Introduction of Cloud Computing into Medical Information Systems
}

\author{
Chen-Lin Lee ${ }^{1,2^{*}}$ \\ 1 Department of Business Administration in Nanhua University, Chia-yi, Taiwan, R.O.C. \\ 2 Senior Supervisor of Buddhist Dalin Tzu-Chi Hospital, Chia-yi, Taiwan, R.O.C. \\ * Corresponding author. Tel.:+886-0933992779; email: allen@tzuchi.org.tw \\ Manuscript submitted May 15,2018;accepted August 14,2018. \\ doi: $10.17706 /$ jcp.13.11.1316-1322
}

\begin{abstract}
Cloud computing has matured and has been widely applied. Managers of medical organizations have noted cloud computing features-computational power, storage capacity, high stability, and flexible design - and have begun to assess the possibility of introducing cloud computing into medical information systems. This study simulated and estimated the costs of introducing cloud computing into medical information systems; the simulation results were compared with the estimated costs of self-maintaining medical information systems to explore the feasibility of introducing cloud computing into medical information systems. This study used mathematical functions to construct two models under the conditions of regular occurrence of damage to information appliances and irregular occurrence of damage to information appliances. Functions of the models were derived and solved; the derived optimal values can serve as references for decision makers.
\end{abstract}

Key words: Cloud computing, medical information systems, mathematical functions.

\section{Introduction}

Cloud technology has been promoted as a tremendous development opportunity for global information technology companies. This technology has been proposed as the next-generation information architecture for enterprises [1]. Cloud computing is an innovative emerging technology that provides benefits such as resource pooling, broad network access, self-service applications [2]-[5] and cost efficiencies [3], [4]. As the market's demand for cloud technology has increased, cloud-related applications have been increasingly prevalent. Cloud computing can be applied for big data operations and for large-scale information processing; additionally, cloud platforms can be used to integrate information systems and develop application software. The majority of sizable enterprises introduce information into cloud platforms to reduce the costs of self-constructed information systems and to control the labor costs of system maintenance. More importantly, they attempt to prevent any local system damage from impairing the operation of the entire information system. Currently, medical organizations mostly rely on internal employees to establish information systems and to conduct maintenance. To maintain and improve the operational effectiveness of the systems, money must be invested in various software and hardware projects; those investments include costs for personnel who perform system maintenance and repair management. Cloud computing systems are stable, capable of fast operation, and equipped with robust storage capacity; the designs of cloud computing systems are flexible. The advantages of cloud computing have gradually attracted numerous managers and researchers in the medical field to introduce cloud 
computing into medical information systems.

The most unique characteristic of cloud computing is its ability to effectively allocate resources and to manage information in a distributed and extensive manner, supporting a variety of platforms, systems, and applications [6]. In studies related to introducing cloud computing into medical information systems, Jin [4] proposed that cloud computing could provide four advantages for medical information architecture-low construction cost, excellent performance, sharing of medical information, and secure data storage. Medical information systems deployed on cloud platforms not only rapidly provide quality service [1] but also improve the efficiency of treatment for patients [7]. The systems can also overcome the obstacle of distance and can enable branch hospitals to exchange data regarding patients' physiological parameters or examination images.

Published research has reported that cloud computing provides stable and flexible conditions for medical information systems and has generated substantial benefits and reduced construction costs. However, few studies have explored the timing of cloud computing rollouts for medical institutions that already have established information systems. Therefore, this study used mathematical models to estimate the costs of self-maintaining the operation of medical information system. In this study's scenario, a hospital had established a medical information system, and the system had been operating continuously; the efficacy and efficiency of medical information appliances were assumed to be unstable and to influence the overall operation. The scenario did not include any medical information systems that were newly developed or had not been established. This study constructed two models based on the following conditions: regular occurrence of damage to information appliances and irregular occurrence of damage to information appliances. The two models were used separately to estimate the cost functions of self-maintaining medical information systems under the two conditions, and the estimated costs were compared with the costs of introducing cloud computing into the system. Hospital decision makers can use these comparisons to understand the costs and benefits of self-maintaining the medical information systems or introducing cloud computing into the information systems under different conditions and restrictions.

\section{Estimating the Costs to Build the Information System}

This study applied mathematical models to estimate the cost function of introducing cloud computing into a medical information system. In the cost function, $R_{j}$ is the risk of loss of the $j$ th information appliance; $L_{j}$ is the loss caused by the risk of loss of the $j$ th information appliance; (1) expresses the fixed cost associated with platform rent and bandwidth expenses after introducing cloud computing into the medical information system. This study also considered the risk factor; that is, $e^{\delta t}$ is the loss transformation rate after the introduction of cloud computing into the medical information system. The mathematical function is as follows:

$$
C_{t}=\int_{0}^{t} \theta+\sum_{j=1}^{m} \alpha\left(R_{j} * L_{j}\right) e^{\delta t} d t
$$

where

$C_{t}$ is the estimated total cost of introducing cloud computing at time point $\mathrm{t}$;

$\theta$ is the fixed expense in the period (i.e., system platform rent plus bandwidth expense);

$\alpha$ is the risk coefficient, $0<\alpha<1$;

$e^{\delta t}$ is the loss transformation rate.

The aforementioned process estimated the costs and risk of loss for introducing cloud computing into a medical information system. For a self-maintained medical information system, in addition to expenditures on essential software and hardware appliances, regular maintenance and the risk of loss due to appliance 
damage should also be considered. For hospital decision makers, if the costs of introducing cloud computing into the medical information system are higher than the estimated costs of self-developing and self-maintaining the information system, the cost would not be a sufficient condition for the introduction of cloud computing. Accordingly, this study analyzed the following two situations: (1) regular occurrence of damage in information appliances and (2) irregular occurrence of damage in information appliances. To determine the models under these two situations and facilitate the search for an optimal solution, this study set $C_{t}$ as $Z$.

\section{Exploration of the Models}

\subsection{The Optimal Solution of Regular Occurrence of Damage in Information Appliances}

The occurrence of risk is assumed to be caused by regular damage to information appliances. Therefore, the total cost $\left(T_{c}\right)$ of self-establishing a medical information system was formalized as (2).

$$
T_{C}=R \lambda L+C(1-R)
$$

where

$R$ is the possibility of damage occurring in information appliances;

$L$ is the risk of loss caused by information appliance damage;

$\lambda$ is the frequency of the damage occurring in information appliances;

$C$ is the cost of establishing the information system.

Under the condition of fixed cost of establishing a medical information system, decision makers in a hospital may seek the optimal approach for reducing the risk of loss to reduce the total loss cost. Therefore, condition (3) was assumed as follows:

$$
\begin{aligned}
\operatorname{Min} T_{C} & =R \lambda L+C(1-R) \\
0 & \leq R \leq 1
\end{aligned}
$$

The optimal solution $R^{*}$ is affected by the value of parameter $\lambda$, that is, $R^{*}=R^{*}(\lambda)$. In practice, decision makers hope to discover $R^{*}$. Once the frequency of failure in information appliances has been reduced, the risk of loss can be reduced. The costs $\left(C_{0}\right)$ generated by appliance failures include parts repair, software function verification and adjustment, and labor costs; other intangible costs include low efficiency and loss of opportunity costs caused by system failures. The intangible costs and other uncontrollable factors were excluded in this study. Preliminary assumptions were tentatively hypothesized regarding costs. All costs were expressed as $C_{0} \lambda \mu+R \lambda L \mu$ during a fixed period such that $\mu$ was the number of information appliances in the hospital. This study assumed $Z$ to be the total expenditure required for introducing cloud computing into the medical information system of the hospital at time $t$; the constraint was that the total costs must not exceed the total budget $Z$ of introducing cloud computing. Therefore, for the condition of $C_{0} \lambda+$ $R \lambda L \leq \frac{Z}{\mu}=\bar{Z}$, the mathematical model was proposed as follows:

$\operatorname{Min} R^{*}(\lambda)$

$$
\text { s. t. } C_{0} \lambda \mu+R \lambda L \mu \leq Z
$$


that is,

$$
C_{0} \lambda+R \lambda L \leq \frac{Z}{\mu} \leq \bar{Z}
$$

Given that the frequency of appliance failure is fixed (i.e., $\lambda$ is a given value), if the objective function $\Pi(R)$ is a linear function, and if the optimal value $R^{*}$ occurs at the endpoint, the optimal value $R^{*}$ has two possible values. When $\lambda \geq \frac{C}{L}$, then $R^{*}=0$; decision makers tend to use self-established and self-maintained information systems. When $\lambda<\frac{C}{L}$, then $R^{*}=1$; decision makers should consider introducing cloud computing into their medical information systems.

$$
R^{*}=\left\{\begin{array}{l}
0 \text { if } \lambda \geq \frac{C}{L} \\
1 \text { if } \lambda<\frac{C}{L}
\end{array}\right\}
$$

Decision makers seek to reduce the frequency of information appliance failures and to reduce the risk of loss. Therefore, given that the introduction of cloud computing is the condition for obtaining the optimal objective value (i.e., $R^{*}=0$ ), the condition is $\lambda \geq \frac{C}{L}$. Therefore, $\lambda \geq \frac{C}{L}$ was substituted into (4) and it was assumed $f(R)=\bar{Z}-C_{0} \lambda-R \lambda L$; when $R^{*}=0$, the function is $f(0)=\bar{Z}-C_{0} \lambda \geq 0$, so that $\lambda \leq \frac{\bar{Z}}{C_{0}}$.

$$
\frac{\bar{Z}}{C_{0}} \geq \lambda \geq \frac{C}{L}
$$

The aforementioned discussions indicate that if the parameters $C_{0}, C, L$, and $\bar{Z}$ satisfy the inequality $\frac{\bar{Z}}{C_{0}} \geq \frac{C}{L}$, and $\lambda$ satisfies the inequality $\frac{\bar{Z}}{C_{0}} \geq \lambda \geq \frac{C}{L}$, then $\bar{Z} \geq \frac{C_{0} C}{L}$.

\subsection{The Optimal Solution of Irregular Occurrence of Damage in Information Appliances}

In a general environment where an information system is operated, the timing of damage occurring in information appliances is uncontrollable. Numerous factors (e.g., temperature and environmental humidity) affect the timing; human factors are also possible causes. In hospitals, people responsible for maintaining the information system cannot fully grasp when the appliances would fail. Any damage might expose the information system to extreme instability, and even though the risks may generate a loss, the maintainers can only attempt to minimize risk passively. Therefore, the larger the $R$ value is, the greater the risk is, leading to larger $\lambda$ and $L$ values. This study assumed that $\lambda(R)$ is a linear function of $R$, and $\lambda(0)$ represents that the information system is operating under stable conditions and does not require repair or replacement of parts; the risk is 0 . Given that $\lambda(R)=K R$, such that $K>0$ is a constant; the mathematical formulas are as follows:

$$
\begin{gathered}
\operatorname{Min} L \lambda(R) R+C(1-R) \\
0 \leq R \leq 1 \\
\operatorname{Min} f(R)=L K R^{2}+C(1-R)
\end{gathered}
$$




$$
0 \leq R \leq 1
$$

This study assumed that $f(R)$ function has a minimum value in the second order derivative function; that is, $f^{\prime \prime}(R)=2 L K \geq 0$. Let $\bar{R}$ be the minimum value of function $f(R)$; then $\bar{R}$ must satisfy the following formula:

$$
f^{\prime}(\bar{R})=2 L K \bar{R}+C \geq 0, \bar{R}=\frac{\mathrm{C}}{2 \mathrm{LK}}
$$

Therefore, $\bar{R}=\frac{C}{2 L K}$ is the lowest point of function $f^{\prime}(\bar{R})$.

\section{Simulation}

\section{The situation of $\bar{R} \leq 1$ was inferred as follows:}

First, let $R^{*}$ be the optimal solution of (5); if $\bar{R} \leq 1, \mathrm{R}^{*}=\bar{R}=\frac{\mathrm{C}}{2 \mathrm{LK}}$; therefore, $R^{*}=\bar{R}=\operatorname{Min}\{\bar{R}, 1\}$;

$R^{*}=\operatorname{Min}\left\{\frac{C}{2 L K}, 1\right\}$ is the optimal risk value that an information system can undertake.

This study assumed $\lambda(R)=K R$, where $K>0$ was a constant. To derive the optimal $\mathrm{K}$ value for decision makers to decide whether to retain the self-maintained medical information system, this study elaborated (2). The condition that the cost cannot exceed the total budget value $\mathrm{Z}$ was retained.

$\operatorname{Min} R^{*}(K)$

$$
\text { s. t. } C_{0} \lambda \mu+R \lambda L \mu \leq Z
$$

$$
\text { that is, } C_{0} K R+R^{2} K L \leq \frac{Z}{\mu} \leq \bar{Z}
$$

$$
\text { from (6), } R^{*}=\operatorname{Min}\left\{\frac{C}{2 L K}, 1\right\}, R^{*}=\bar{R}=\frac{C}{2 L K} \leq 1
$$

$$
\text { that is, } K \geq \frac{C}{2 L} \text {, }
$$

let $f(R)=\bar{Z}-C_{0} K R-R^{2} K L$, and substitute $R^{*}=\frac{C}{2 L K}$ into (7),

$$
\begin{gathered}
\bar{Z}-C_{0} K \frac{C}{2 L K}-\left(\frac{C}{2 L K}\right)^{2} K L \geq 0 \\
\bar{Z}-\frac{C_{0} C}{2 L}-\frac{C^{2}}{4 L K} \geq 0 \\
\frac{C_{0} C}{2 L}+\frac{C^{2}}{4 L K} \leq \bar{Z}
\end{gathered}
$$

In Fig. 1, $K$ can be any value as long as it satisfies $K \geq \frac{C}{2 L}$; therefore, if $\frac{C^{2}}{4 L K}$ equals 0 , then $\bar{Z} \geq \frac{C_{0} C}{2 L}$. When $K<\frac{C}{2 L}$, the decision makers bear the maximal risk of loss. Under this situation, the decision makers encounter extreme instability of the information appliances. When $K \geq \frac{C}{2 L}$, the risk borne by decision makers begins to decrease and gradually reaches stable status. 


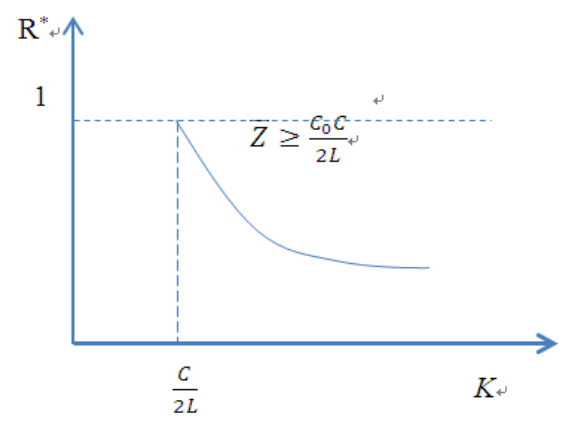

Fig. 1. Determination of a K value.

To obtain the optimal $K$, this study simplified (9) and derived (10) as follows:

$$
K \leq \frac{C^{2}}{2 C_{0} C-4 L \bar{Z}}
$$

After the integration of inequalities (8) and (10), the optimal $K$ value must satisfy the following condition:

$$
\frac{C^{2}}{2 C_{0} C-4 L \bar{Z}} \geq K \geq \frac{C}{2 L}
$$

The inequality shows that if parameters $C_{0}, C, L$, and $\bar{Z}$ satisfy the inequality, it can be rewritten as follows:

$$
\frac{C^{2}}{2 C_{0} C-4 L \bar{Z}} \geq \frac{C}{2 L}
$$

If decision makers of the hospital choose some $K$ value that satisfies the inequality $\frac{C^{2}}{2 C_{0} C-4 L \bar{Z}} \geq K \geq \frac{C}{2 L}$, this indicates that the risk $R^{*}$ can be effectively minimized. The inequality also means $\frac{C^{2}}{2 C_{0} C-4 L \bar{Z}} \geq \frac{C}{2 L}$; given any $K$ value associated with high risk of loss of the medical information system, the decision makers are recommended not to introduce cloud computing into the medical information system.

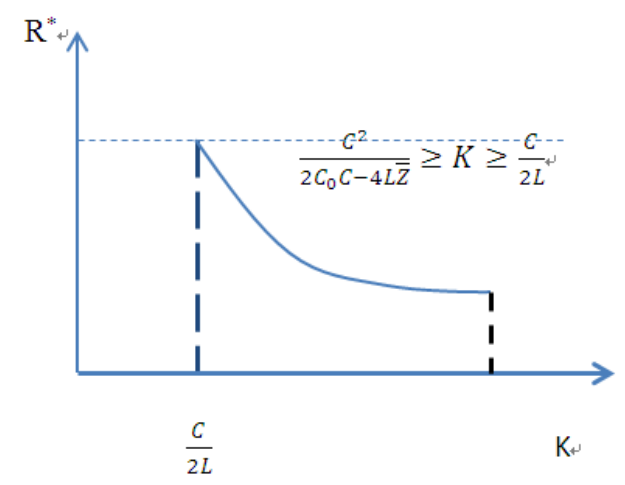

Fig. 2. Determination of constraints for K value.

\section{Conclusion}

This paper analyzes and discusses different models of medical information systems from the perspectives of hospital decision makers. Two models are proposed as references for decision makers to determine whether the hospital should self-maintain the medical information system or introduce cloud computing into the system. Self-maintaining medical information systems constitute a typical measure implemented by most medical institutions. The models provide decision makers with different perspectives on determining whether medical information systems should be self-maintained, or cloud computing should be introduced. 


\section{References}

[1] Arunkumar, R. J., \& Anbuselvi, R. (2017). Enhancement of cloud computing security in health care sector. International Journal of Computer Science and Mobile Computing, 16(8), 23-31.

[2] Ratten, V. (2016). Continuance use intention of cloud computing: Innovativeness and creativity perspectives. Journal of Business Research, 69, 1737-1740.

[3] Ratten, V. (2015). International consumer attitudes toward cloud computing: A social cognitive theory and technology acceptance model perspective. Thunderbird International Business Review, 57(3), 217-228.

[4] Jin, H. (2017). Application discussion of cloud computing in hospital information construction. Journal of Applied Science Engineering Innovation, 4(4), 144-147.

[5] Son, I., Lee, D., Lee, J., \& Chang, Y. (2014). Market perception on cloud computing initiatives in organizations: An extended resource-base view. Information \& Management, 51, 653-669.

[6] Poonam, B., Sutar, B., \& Kulkarni, P. (2012). Cloud computing support for enhanced health applications. International Journal of Engineering and Innovative Technology, 2(1), 139-141.

Raval, D., \& Jangale, S. (2016). Cloud based information security and privacy in healthcare. International Journal of Computer Applications, 150(4), 11-15.

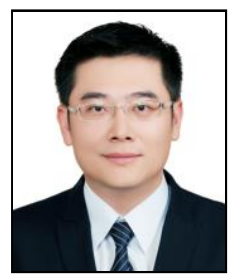

Chen-Lin Lee is an assistant professor of Department of Business Administration at Nanhua University in Taiwan. He also is a senior supervisor of Buddhist Dalin Tzu-Chi Hospital. His research interests are in the area of medical information management, Modeling and computational intelligence. 